\title{
Physiological changes following transplantation of the bivalve Scrobicularia plana between three populations
}

\author{
C. M. Worrall and J. Widdows \\ Institute for Marine Environmental Research, Prospect Place, The Hoe, Plymouth PL1 3DH, United Kingdom
}

\begin{abstract}
Transplants of the bivalve Scrobicularia plana (Da Costa) were made between the Lynher East Looe and Gannel estuaries (South West England) to ascertain the plasticity of the physiological responses recorded in the native populations. All transplants showed either a partial or almost complete acclimatization to conditions at the transplant site which indicated that the physiological differences between populations were largely environmentally induced, i.e. phenotypic. Incomplete acclimatization could be attributed to either the relatively short transplant period of 3 mo or the timing of the transplant in relation to the reproductive cycle.
\end{abstract}

\section{INTRODUCTION}

In a preceding paper, Worrall et al. (1983) recorded significant physiological differences between populations of the bivalve Scrobicularia plana from 3 estuaries in S.W. England - Lynher, E. Looe and Gannel. These differ markedly in their sediment food levels (Worrall et al., 1983) and their sediment heavy metal concentrations (Bryan and Hummerstone, 1978; Worrall et al., in prep).

In this paper, we compare the physiological responses of transplanted Scrobicularia plana with those of native individuals from the 3 estuaries. Reciprocal transplants were carried out in order to examine the plasticity of the physiological response and the extent to which measured physiological differences in the native populations may be environmentally induced (i.e. phenotypic) rather than genetically determined characteristics of the populations (i.e. genotypic).

Transplantation techniques have been used in several field studies (Phelps et al., 1981; Widdows et al.,

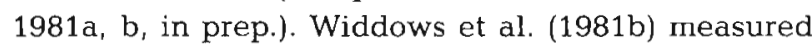
the effects of a pollution gradient in Narragansett Bay, Rhode Island (USA), on the physiological condition of transplanted mussels Mytilus edulis. Widdows et al., (in prep.) have carried out reciprocal transplants between 2 physiologically different populations of $M$. edulis. Bivalves have also been transplanted to diffe- rent environments in order to examine uptake and depuration of heavy metals by body tissues (Scrobicularia plana - Bryan and Hummerstone, 1978; M. edulis - Simpson, 1979; Mercenaria mercenaria Behrens and Duedall, 1981).

\section{MATERIAL AND METHODS}

Scrobicularia plana were collected from the intertidal zone at low water spring tides from 3 estuaries in Cornwall (S.W. England), the Lynher, E. Looe and Gannel. Two sets of $S$. plana transplants were carried out for a duration of 3 mo between Lynher and E. Looe (beginning April 1978 and December 1978), between Lynher and Gannel (beginning August 1978 and April 1979), and between E. Looe and Gannel (beginning June 1978 and February 1979). Individual bivalves (120) were marked on each shell valve with enamel paint and were introduced to the sediment of the new environment without caged boundaries but within a marked area $\left(1 \mathrm{~m}^{2}\right)$ and ensuring that the native density (no. $\mathrm{m}^{-2}$ ) was maintained. After $3 \mathrm{mo}$ at the new site, transplanted bivalves were removed from the sediment and returned to the laboratory for physiological measurements; at the same time a sample of surface sediment was taken for particulate analysis (for details see Worrall et al., 1983). 


\section{RESULTS AND DISCUSSION}

Physiological measurements were carried out on 3 native Scrobicularia plana populations and significant differences were recorded (Worrall et al., 1983). Two sets of reciprocal transplants between the sites were carried out for a duration of $3 \mathrm{mo}$, to examine the plasticity of the physiological responses and the relative importance of environmental factors in determining the observed physiological differences.

The time of transplantation together with the bivalve's condition at the start of the transplant were important in subsequent recovery and performance. Recovery of Scrobicularia plana can be defined as the number of individuals recaptured alive and in good physiological condition. Several marked shells of dead bivalves were recovered from the 3 sites but mortality estimates could not be made because dead shells could be lost from the substrate due to tidal action since bivalves were not caged within the sediment. Recovery was greater if transplants were made during winter when gametogenesis was dormant but was much lower when transplants were made during summer when bivalves contained ripe gametes or were in a poor condition after spawning (Table 1).

Table 1. Scrobicularia plana. Percentage recovery after a 3 mo transplant between sites $(n=120) . L=L y n h e r, E L=E$. Looe,$\quad G=$ Gannel,$\quad \mathrm{L} \rightarrow \mathrm{EL}=$ Lynher individuals transplanted to the E. Looe site

\begin{tabular}{|cccc}
\cline { 1 - 3 } Transplant & $\begin{array}{c}\text { Date } \\
\text { transplanted }\end{array}$ & $\begin{array}{c}\text { Date } \\
\text { recovered }\end{array}$ & $\begin{array}{c}\% \\
\text { recovery }\end{array}$ \\
\hline $\mathrm{L} \rightarrow \mathrm{EL}$ & 12 Dec 1977 & 19 Mar 1978 & 27 \\
$\mathrm{EL} \rightarrow \mathrm{L}$ & 12 Dec 1977 & 12 Mar 1978 & 23 \\
$\mathrm{~L} \rightarrow \mathrm{EL}$ & 12 Apr 1978 & 7 Jul 1978 & 13 \\
$\mathrm{EL} \rightarrow \mathrm{L}$ & 12 Apr 1978 & 17 Jul 1978 & 32 \\
$\mathrm{EL} \rightarrow \mathrm{G}$ & 14 Jun 1978 & 4 Sep 1978 & 27 \\
$\mathrm{G} \rightarrow \mathrm{EL}$ & 14 Jun 1978 & 18 Sep 1978 & 10 \\
$\mathrm{EL} \rightarrow \mathrm{G}$ & 1 Feb 1979 & 14 May 1979 & 61 \\
$\mathrm{G} \rightarrow \mathrm{EL}$ & 1 Feb 1979 & 21 May 1979 & 36 \\
$\mathrm{G} \rightarrow \mathrm{L}$ & 11 Aug 1978 & 13 Nov 1978 & 9 \\
$\mathrm{~L} \rightarrow \mathrm{G}$ & 11 Aug 1978 & 27 Nov 1978 & 9 \\
$\mathrm{G} \rightarrow \mathrm{L}$ & 28 Mar 1979 & 16 Jul 1979 & 43 \\
$\mathrm{~L} \rightarrow \mathrm{G}$ & 28 Mar1979 & 9 Jul 1979 & 43 \\
& & & \\
\end{tabular}

Measurements of oxygen consumption, clearance rate, ammonia excretion, oxygen: nitrogen ratio, scope for growth, convection requirement and length/weight

Table 2. First transplant series. Summary of the physiological data of Scrobicularia plana transplants. L $=\mathrm{Lynher}$, EL $=\mathrm{E}$. Looe, $\mathrm{G}=$ Gannel, $\mathrm{N}=$ native individuals. $\mathrm{L} \rightarrow \mathrm{EL}=$ Lynher individuals transplanted to the $\mathrm{E}$. Looe population. All measurements are corrected to a standard individual of $100 \mathrm{mg}$ dry weight. $t_{0}=$ start of transplant, $t_{3 m}=$ end of transplant 3 mo later

\begin{tabular}{|c|c|c|c|c|c|c|}
\hline \multirow{2}{*}{$\begin{array}{l}\text { Physiological } \\
\text { parameters }\end{array}$} & \multicolumn{2}{|c|}{ Lynher $\rightleftarrows$ E. Looe transplants } & \multicolumn{2}{|c|}{ E. Looe $\rightleftarrows$ Gannel transplants } & \multicolumn{2}{|c|}{ Gannel $\rightleftarrows$ Lynher transplants } \\
\hline & $\begin{array}{c}\text { April } 1978 \\
\left(t_{0}\right)\end{array}$ & $\begin{array}{l}\text { July } 1978 \\
\left(t_{3 m}\right)\end{array}$ & $\begin{array}{c}\text { June } 1978 \\
\left(t_{0}\right)\end{array}$ & $\begin{array}{l}\text { September } 1978 \\
\left(t_{3 \mathrm{~m}}\right)\end{array}$ & $\begin{array}{c}\text { August } 1978 \\
\left(\mathrm{t}_{\circ}\right)\end{array}$ & $\begin{array}{c}\text { November } 1978 \\
\left(t_{3 \mathrm{~m}}\right)\end{array}$ \\
\hline $\begin{array}{l}\text { Oxygen consumption } \\
\left(\mu \mathrm{l} \mathrm{O}_{2} \mathrm{~h}^{-1}\right)\end{array}$ & $\begin{aligned} \mathrm{LN} & =11 \\
\mathrm{ELN} & =11\end{aligned}$ & $\begin{aligned} \mathrm{LN} & =31 \\
\mathrm{ELN} & =25 \\
\mathrm{~L} \rightarrow \mathrm{EL} & =35 \\
\mathrm{EL} \rightarrow \mathrm{L} & =26\end{aligned}$ & $\begin{aligned} \mathrm{ELN} & =25 \\
\mathrm{GN} & =29\end{aligned}$ & $\begin{aligned} \mathrm{ELN} & =25 \\
\mathrm{GN} & =24 \\
\mathrm{EL} \rightarrow \mathrm{G} & =21 \\
\mathrm{G} \rightarrow \mathrm{EL} & =29\end{aligned}$ & $\begin{array}{l}\mathrm{LN}=25 \\
\mathrm{GN}=24\end{array}$ & $\begin{aligned} \mathrm{LN} & =19 \\
\mathrm{GN} & =17 \\
\mathrm{~L} \rightarrow \mathrm{G} & =23 \\
\mathrm{G} \rightarrow \mathrm{L} & =18\end{aligned}$ \\
\hline $\begin{array}{l}\text { Clearance rate } \\
\left(\mathrm{ml} \mathrm{h}^{-1}\right)\end{array}$ & $\begin{aligned} \mathrm{LN} & =36 \\
\mathrm{ELN} & =27\end{aligned}$ & $\begin{aligned} \mathrm{LN} & =52 \\
\mathrm{ELN} & =72 \\
\mathrm{~L} \rightarrow \mathrm{EL} & =60 \\
\mathrm{EL} \rightarrow \mathrm{L} & =70\end{aligned}$ & $\begin{aligned} \mathrm{ELN} & =72 \\
\mathrm{GN} & =90\end{aligned}$ & $\begin{aligned} \mathrm{ELN} & =45 \\
\mathrm{GN} & =100 \\
\mathrm{EL} \rightarrow \mathrm{G} & =47 \\
\mathrm{G} \rightarrow \mathrm{EL} & =75\end{aligned}$ & $\begin{aligned} \mathrm{LN} & =54 \\
\mathrm{GN} & =100\end{aligned}$ & $\begin{aligned} \mathrm{LN} & =70 \\
\mathrm{GN} & =102 \\
\mathrm{~L} \rightarrow \mathrm{G} & =56 \\
\mathrm{G} \rightarrow \mathrm{L} & =44\end{aligned}$ \\
\hline $\begin{array}{l}\text { Ammonia excretion } \\
\left(\mu \mathrm{g} \mathrm{NH}_{4} \mathrm{~N} \mathrm{~h}^{-1}\right)\end{array}$ & $\begin{aligned} \mathrm{LN} & =0.21 \\
\mathrm{ELN} & =0.32\end{aligned}$ & $\begin{aligned} \mathrm{LN} & =1.12 \\
\mathrm{ELN} & =1.25 \\
\mathrm{~L} \rightarrow \mathrm{EL} & =1.84 \\
\mathrm{EL} \rightarrow \mathrm{L} & =1.02\end{aligned}$ & $\begin{aligned} \mathrm{ELN} & =1.25 \\
\mathrm{GN} & =0.42\end{aligned}$ & $\begin{aligned} \mathrm{ELN} & =1.20 \\
\mathrm{GN} & =1.56 \\
\mathrm{EL} \rightarrow \mathrm{G} & =1.52 \\
\mathrm{G} \rightarrow \mathrm{EL} & =1.70\end{aligned}$ & $\begin{aligned} \mathrm{LN} & =1.30 \\
\mathrm{GN} & =1.56\end{aligned}$ & $\begin{aligned} \mathrm{LN} & =0.86 \\
\mathrm{GN} & =0.53 \\
\mathrm{~L} \rightarrow \mathrm{G} & =1.98 \\
\mathrm{G} \rightarrow \mathrm{L} & =1.65\end{aligned}$ \\
\hline $\mathrm{O}: \mathrm{N}$ ratio & $\begin{aligned} \mathrm{LN} & =68.4 \\
\mathrm{ELN} & =45.8\end{aligned}$ & $\begin{aligned} \mathrm{LN} & =34.6 \\
\mathrm{ELN} & =25.1 \\
\mathrm{~L} \rightarrow \mathrm{EL} & =23.8 \\
\mathrm{EL} \rightarrow \mathrm{L} & =32.0\end{aligned}$ & $\begin{aligned} \mathrm{ELN} & =25.1 \\
\mathrm{GN} & =86.3\end{aligned}$ & $\begin{aligned} \mathrm{ELN} & =26.1 \\
\mathrm{GN} & =19.3 \\
\mathrm{EL} \rightarrow \mathrm{G} & =17.6 \\
\mathrm{G} \rightarrow \mathrm{EL} & =21.4\end{aligned}$ & $\begin{aligned} \mathrm{LN} & =24.1 \\
\mathrm{GN} & =19.3\end{aligned}$ & $\begin{aligned} \mathrm{LN} & =27.7 \\
\mathrm{GN} & =39.6 \\
\mathrm{~L} \rightarrow \mathrm{G} & =14.7 \\
\mathrm{G} \rightarrow \mathrm{L} & =13.8\end{aligned}$ \\
\hline $\begin{array}{l}\text { Dry weight of a } 3 \mathrm{~cm} \\
\text { individual ( } \mathrm{mg} \text { ) }\end{array}$ & $\begin{aligned} \mathrm{LN} & =160 \\
\mathrm{ELN} & =150\end{aligned}$ & $\begin{aligned} \mathrm{LN} & =200 \\
\mathrm{ELN} & =165 \\
\mathrm{~L} \rightarrow \mathrm{EL} & =150 \\
\mathrm{EL} \rightarrow \mathrm{L} & =185\end{aligned}$ & $\begin{aligned} \mathrm{ELN} & =160 \\
\mathrm{GN} & =98\end{aligned}$ & $\begin{aligned} \mathrm{ELN} & =175 \\
\mathrm{GN} & =94 \\
\mathrm{EL} \rightarrow \mathrm{G} & =162 \\
\mathrm{G} \rightarrow \mathrm{EL} & =125\end{aligned}$ & $\begin{aligned} \mathrm{LN} & =180 \\
\mathrm{GN} & =94\end{aligned}$ & $\begin{aligned} \mathrm{LN} & =192 \\
\mathrm{GN} & =115 \\
\mathrm{~L} \rightarrow \mathrm{G} & =127 \\
\mathrm{G} \rightarrow \mathrm{L} & =130\end{aligned}$ \\
\hline $\begin{array}{l}\text { Convection requirement } \\
\mathrm{CR} / \mathrm{VO}_{2}\left(\text { litres } \mathrm{ml} \mathrm{O} \mathrm{O}^{-1} \text { ) }\right.\end{array}$ & $\begin{aligned} \mathrm{LN} & =3.18 \\
\mathrm{ELN} & =2.37\end{aligned}$ & $\begin{aligned} \mathrm{LN} & =1.68 \\
\mathrm{ELN} & =2.88 \\
\mathrm{~L} \rightarrow \mathrm{EL} & =1.70 \\
\mathrm{EL} \rightarrow \mathrm{L} & =2.69\end{aligned}$ & $\begin{aligned} E L N & =2.88 \\
G N & =3.10\end{aligned}$ & $\begin{aligned} \mathrm{ELN} & =1.80 \\
\mathrm{GN} & =4.17 \\
\mathrm{EL} \rightarrow \mathrm{G} & =2.24 \\
\mathrm{G} \rightarrow \mathrm{EL} & =2.59\end{aligned}$ & $\begin{aligned} \mathrm{LN} & =2.16 \\
\mathrm{GN} & =4.17\end{aligned}$ & $\begin{aligned} \mathrm{LN} & =3.68 \\
\mathrm{GN} & =6.07 \\
\mathrm{~L} \rightarrow \mathrm{G} & =2.42 \\
\mathrm{G} \rightarrow \mathrm{L} & =2.39\end{aligned}$ \\
\hline
\end{tabular}


Table 3. Second transplant series. Summary of the physiological data of Scrobicularia plana transplants. See legend to Table 2 for details. 'E. Looe native measurements taken in late Febmary, $\bullet$. Looe native measurements taken in April, oLynher native measurements taken in June

\begin{tabular}{|c|c|c|c|c|c|c|}
\hline \multirow{2}{*}{$\begin{array}{l}\text { Physiological } \\
\text { parameters }\end{array}$} & \multicolumn{2}{|c|}{ Lynher $\rightleftarrows$ E. Looe transplants } & \multicolumn{2}{|c|}{ E. Looe $\rightleftarrows$ Gannel transplants } & \multicolumn{2}{|c|}{ Gannel $\rightleftarrows$ Lynher transplants } \\
\hline & $\begin{array}{l}\text { December } \\
1978\left(\mathrm{t}_{\mathrm{o}}\right)\end{array}$ & $\begin{array}{l}\text { March } 1979 \\
\quad\left(t_{3 m}\right)\end{array}$ & $\begin{array}{l}\text { February } \\
1979\left(t_{o}\right)\end{array}$ & $\begin{array}{l}\text { May } 1979 \\
\quad\left(\mathrm{t}_{3 \mathrm{~m}}\right)\end{array}$ & $\begin{array}{l}\text { April } 1979 \\
\left(t_{0}\right)\end{array}$ & $\begin{array}{l}\text { July } 1979 \\
\qquad\left(t_{3 m}\right)\end{array}$ \\
\hline $\begin{array}{l}\text { Oxygen consumption } \\
\left(\mathrm{\mu l} \mathrm{O}_{2} \mathrm{~h}^{-1}\right)\end{array}$ & $\begin{aligned} \mathrm{LN} & =21 \\
\mathrm{ELN} & =19\end{aligned}$ & $\begin{aligned} \mathrm{LN} & =13 \\
\cdot \mathrm{ELN} & =11 \\
\mathrm{~L} \rightarrow \mathrm{EL} & =6 \\
\mathrm{EL} \rightarrow \mathrm{L} & =9\end{aligned}$ & $\begin{aligned} \mathrm{ELN} & =11 \\
\mathrm{GN} & =11\end{aligned}$ & $\begin{aligned} \bullet \mathrm{ELN} & =15 \\
\mathrm{GN} & =16 \\
\mathrm{EL} \rightarrow \mathrm{G} & =13 \\
\mathrm{G} \rightarrow \mathrm{EL} & =17\end{aligned}$ & $\begin{aligned} \mathrm{LN} & =13 \\
\mathrm{GN} & =16\end{aligned}$ & $\begin{aligned} \mathrm{OLN} & =19 \\
\mathrm{GN} & =17 \\
\mathrm{~L} \rightarrow \mathrm{G} & =15 \\
\mathrm{G} \rightarrow \mathrm{L} & =21\end{aligned}$ \\
\hline $\begin{array}{l}\text { Clearance rate } \\
\left(\mathrm{ml} \mathrm{h}^{-1}\right)\end{array}$ & $\begin{aligned} \mathrm{LN} & =45 \\
\mathrm{ELN} & =90\end{aligned}$ & $\begin{aligned} \mathrm{LN} & =25 \\
\cdot \mathrm{ELN} & =35 \\
\mathrm{~L} \rightarrow \mathrm{EL} & =23 \\
\mathrm{EL} \rightarrow \mathrm{L} & =52\end{aligned}$ & $\begin{aligned} \mathrm{ELN} & =35 \\
\mathrm{GN} & =44\end{aligned}$ & $\begin{aligned} \bullet \mathrm{ELN} & =44 \\
\mathrm{GN} & =56 \\
\mathrm{EL} \rightarrow \mathrm{G} & =67 \\
\mathrm{G} \rightarrow \mathrm{EL} & =81\end{aligned}$ & $\begin{aligned} \mathrm{LN} & =25 \\
\mathrm{GN} & =56\end{aligned}$ & $\begin{aligned} \mathrm{OLN} & =56 \\
\mathrm{GN} & =76 \\
\mathrm{~L} \rightarrow \mathrm{G} & =63 \\
\mathrm{G} \rightarrow \mathrm{L} & =80\end{aligned}$ \\
\hline $\begin{array}{l}\text { Ammonia excretion } \\
\left(\mu \mathrm{g} \mathrm{NH}{ }_{4} \mathrm{Nh}^{-1}\right)\end{array}$ & $\begin{aligned} \mathrm{LN} & =1.58 \\
\mathrm{ELN} & =1.01\end{aligned}$ & $\begin{aligned} \mathrm{LN} & =0.09 \\
\cdot \mathrm{ELN} & =1.13 \\
\mathrm{~L} \rightarrow \mathrm{EL} & =0.14 \\
\mathrm{EL} \rightarrow \mathrm{L} & =0.79\end{aligned}$ & $\begin{aligned} \mathrm{ELN} & =1.13 \\
\mathrm{GN} & =0.64\end{aligned}$ & $\begin{aligned}-\mathrm{ELN} & =0.52 \\
\mathrm{GN} & =0.52 \\
\mathrm{EL} \rightarrow \mathrm{G} & =0.61 \\
\mathrm{G} \rightarrow \mathrm{EL} & =0.43\end{aligned}$ & $\begin{aligned} \mathrm{LN} & =0.09 \\
\mathrm{GN} & =0.52\end{aligned}$ & $\begin{aligned} \mathrm{OLN} & =1.21 \\
\mathrm{GN} & =0.57 \\
\mathrm{~L} \rightarrow \mathrm{G} & =0.53 \\
\mathrm{G} \rightarrow \mathrm{L} & =0.89\end{aligned}$ \\
\hline$O:$ Nratio & $\begin{aligned} \mathrm{LN} & =16.9 \\
\mathrm{ELN} & =23.6\end{aligned}$ & $\begin{aligned} \mathrm{LN} & =174.6 \\
\cdot \mathrm{ELN} & =11.7 \\
\mathrm{~L} \rightarrow \mathrm{EL} & =51.8 \\
\mathrm{EL} \rightarrow \mathrm{L} & =14.6\end{aligned}$ & $\begin{aligned} E L N & =11.7 \\
G N & =21.9\end{aligned}$ & $\begin{aligned} \bullet \mathrm{ELN} & =35.9 \\
\mathrm{GN} & =38.5 \\
\mathrm{EL} \rightarrow \mathrm{G} & =26.5 \\
\mathrm{G} \rightarrow \mathrm{EL} & =50.4\end{aligned}$ & $\begin{aligned} \mathrm{LN} & =174.6 \\
\mathrm{GN} & =38.5\end{aligned}$ & $\begin{aligned} \mathrm{LNN} & =20.2 \\
\mathrm{GN} & =38.3 \\
\mathrm{~L} \rightarrow \mathrm{G} & =36.2 \\
\mathrm{G} \rightarrow \mathrm{L} & =30.0\end{aligned}$ \\
\hline $\begin{array}{l}\text { Dry weight of a } 3 \mathrm{~cm} \\
\text { individual }(\mathrm{mg})\end{array}$ & $\begin{aligned} \mathrm{LN} & =167 \\
\mathrm{ELN} & =148\end{aligned}$ & $\begin{aligned} \mathrm{LN} & =170 \\
\cdot \mathrm{ELN} & =155 \\
\mathrm{~L} \rightarrow \mathrm{EL} & =172 \\
\mathrm{EL} \rightarrow \mathrm{L} & =158\end{aligned}$ & $\begin{aligned} E L N & =155 \\
G N & =106\end{aligned}$ & $\begin{aligned} \cdot \mathrm{ELN} & =165 \\
\mathrm{GN} & =123 \\
\mathrm{EL} \rightarrow \mathrm{G} & =130 \\
\mathrm{G} \rightarrow \mathrm{EL} & =113\end{aligned}$ & $\begin{aligned} \mathrm{LN} & =170 \\
\mathrm{GN} & =123\end{aligned}$ & $\begin{aligned} \mathrm{OLN} & =163 \\
\mathrm{GN} & =108 \\
\mathrm{~L} \rightarrow \mathrm{G} & =147 \\
\mathrm{G} \rightarrow \mathrm{L} & =180\end{aligned}$ \\
\hline $\begin{array}{l}\text { Convection requirement } \\
\mathrm{CR} \sim \mathrm{VO}_{2}\left(\text { litres } \mathrm{ml} \mathrm{O}_{2}^{-1} \text { ) }\right.\end{array}$ & $\begin{aligned} \mathrm{LN} & =2.10 \\
\mathrm{ELN} & =4.74\end{aligned}$ & $\begin{aligned} \mathrm{LN} & =1.97 \\
\cdot \mathrm{ELN} & =3.25 \\
\mathrm{~L} \rightarrow \mathrm{EL} & =3.97 \\
\mathrm{EL} \rightarrow \mathrm{L} & =5.65\end{aligned}$ & $\begin{aligned} \mathrm{ELN} & =3.25 \\
\mathrm{GN} & =3.96\end{aligned}$ & $\begin{aligned} \bullet \mathrm{ELN} & =2.97 \\
\mathrm{GN} & =3.50 \\
\mathrm{EL} \rightarrow \mathrm{G} & =5.23 \\
\mathrm{G} \rightarrow \mathrm{EL} & =4.68\end{aligned}$ & $\begin{aligned} \mathrm{LN} & =1.97 \\
\mathrm{GN} & =3.50\end{aligned}$ & $\begin{aligned} \circ \mathrm{LN} & =2.88 \\
\mathrm{GN} & =4.37 \\
\mathrm{~L} \rightarrow \mathrm{G} & =4.14 \\
\mathrm{G} \rightarrow \mathrm{L} & =3.77\end{aligned}$ \\
\hline
\end{tabular}

relationship were made on the 2 native Scrobicularia plana populations at the beginning and end of the transplant period, and both sets of transplanted bivalves were measured after 3 mo. All measurements listed in Tables 2 and 3 were corrected to a standard individual size of $100 \mathrm{mg}$ dry flesh weight.

The relationship between dry flesh weight and physiological responses of native and transplanted Scrobicularia plana from the 3 sites (Tables 2 and 3) were analysed by regression and covariance techniques following $\log _{10}$ transformation. The F-ratio in the analysis of variance was calculated to determine whether there was a significant difference in gradients, and if not, whether there was a significant difference between means. All physiological measurements listed in Tables 4 and 5 are those which show significant differences between means at $\mathrm{P}<0.05$.

Scrobicularia plana transplanted from Lynher to E. Looe (April-June 1978) showed a reduction in dry flesh weight, an increase in ammonia excretion and a decline in the $\mathrm{O}: \mathrm{N}$ ratio, indicating an overall decline in condition (Table 2). In contrast, the bivalves transplanted from E. Looe to Lynher showed the reverse with an overall improvement in physiological condition. When Lynher and E. Looe bivalves were transplanted to Gannel there were some marked responses to the change in environment. In both transplants to the Gannel there was a decline in dry flesh weight and $O: N$ ratio, but no increase in clearance rate towards the higher rate of the Gannel natives. S. plana transplanted from Gannel to Lynher and E. Looe, however, generally increased in tissue weight and reduced clearance rate towards that of the native populations.

In the second series of transplants (Table 3 ) bivalves transferred from Lynher (April-July 1979) and E. Looe (February-May 1979) to the Gannel showed a decline in oxygen consumption, ammonia excretion and tissue weight but an increase in clearance rate towards the Gannel population. In contrast, Gannel individuals transplanted to either Lynher or E. Looe showed an increase in oxygen uptake and a maintained or increased clearance rate. The Gannel individuals at the Lynher site increased tissue weight even relative to the Lynher natives which showed a slight decline possibly due to early spawning. Scope for growth values for native and transplanted Scrobicularia plana are 
Table 4. First transplant series. Comparison of native and transplanted Scrobicularia plana at Lynher (L), E. Looe (EL) and Gannel (G) sites. Physiological measurements were analysed by regression and covariance techniques following log ${ }_{10}$ transformation. $\mathrm{N}=$ native individuals, LN vs. ELN compares physiological measurements of Lynher and E. Looe native individuals , $\mathrm{L} \rightarrow \mathrm{EL}$ vs. LN compares the physiological responses of Lynher individuals transplanted to E. Looe with the Lynher native's response. The physiological parameters measured include oxygen consumption (VO $)_{2}$, clearance rate (CR), ammonia excretion $\left(\mathrm{NH}_{4}\right)$ and length-weight analysis (LW). The listing refers to the number of physiological measurements that are significant at

$\mathrm{P}<0.05 . \mathrm{t}=0$ is time that the transplant started, and $\mathrm{t}=3 \mathrm{~m}$ time that the transplant ended after 3 mo at the new site

\begin{tabular}{|c|c|c|c|}
\hline Comparison & Date and time & $\begin{array}{l}\text { List of statistically } \\
\text { significant population } \\
\text { differences in } \\
\text { physiological responses }\end{array}$ & General assessment \\
\hline $\begin{array}{l}\text { LN vs. ELN } \\
\text { LN vs. ELN }\end{array}$ & $\begin{array}{l}\text { Apr 1978, } t=0 \\
\text { Jun 1978,t }=3 \mathrm{~m}\end{array}$ & $\begin{array}{ll}3 & \left(\mathrm{CR}, \mathrm{NH}_{4}, \mathrm{LW}\right) \\
2 & (\mathrm{CR}, \mathrm{LW})\end{array}$ & Population differences \\
\hline $\begin{array}{l}\mathrm{L} \rightarrow \mathrm{EL} \text { vs. } \mathrm{LN} \\
\mathrm{L} \rightarrow \mathrm{EL} \text { vs. ELN } \\
\mathrm{EL} \rightarrow \mathrm{L} \text { vs. ELN } \\
\mathrm{EL} \rightarrow \mathrm{L} \text { vs. } \mathrm{LN}\end{array}$ & $\begin{array}{l}\text { Jun } 1978, t=3 m \\
\text { Jun } 1978, t=3 m \\
\text { Jun } 1978, t=3 m \\
\text { Jun } 1978, t=3 m\end{array}$ & $\begin{array}{ll}2 & (\mathrm{CR}, \mathrm{LW}) \\
2 & \left(\mathrm{VO}_{2}, \mathrm{CR}\right) \\
2 & \left(\mathrm{NH}_{4}, \mathrm{LW}\right) \\
1 & (\mathrm{LW})\end{array}$ & $\begin{array}{l}\text { Population differences partly maintained } \\
\text { Little environmental adaptation to E. Looe } \\
\text { Adaptation to Lynher almost complete }\end{array}$ \\
\hline $\begin{array}{l}\text { ELN vs. GN } \\
\text { ELN vs. GN }\end{array}$ & $\begin{array}{l}\text { Jun } 1978, t=o \\
\text { Sep } 1978, t=3 \mathrm{~m}\end{array}$ & $\begin{array}{ll}2 & \left(\mathrm{NH}_{4}, \mathrm{LW}\right) \\
3 & \left(\mathrm{NH}_{4}, \mathrm{CR}, \mathrm{LW}\right)\end{array}$ & Population differences \\
\hline $\begin{array}{l}\mathrm{EL} \rightarrow \mathrm{G} \text { vs. ELN } \\
\mathrm{EL} \rightarrow \mathrm{G} \text { vs. GN } \\
\mathrm{G} \rightarrow \mathrm{EL} \text { vs. GN } \\
\mathrm{G} \rightarrow \mathrm{EL} \text { vs. ELN }\end{array}$ & $\begin{array}{l}\text { Sep } 1978, t=3 m \\
\text { Sep 1978,t }=3 m \\
\text { Sep } 1978, t=3 m \\
\text { Sep } 1978, t=3 m\end{array}$ & $\begin{array}{ll}4 & \left(\mathrm{CR}, \mathrm{VO}_{2}, \mathrm{NH}_{4}, \mathrm{LW}\right) \\
2 & (\mathrm{CR}, \mathrm{LW}) \\
1 & (\mathrm{LW}) \\
3 & \left(\mathrm{CR}, \mathrm{NH}_{4}, \mathrm{LW}\right)\end{array}$ & $\begin{array}{l}\text { Environmental adaptation to Gannel } \\
\text { Population differences partly maintained } \\
\text { Little adaptation to E. Looe }\end{array}$ \\
\hline $\begin{array}{l}\text { GN vs. LN } \\
\text { GN vs. LN }\end{array}$ & $\begin{array}{l}\text { Aug } 1978, \mathrm{t}=\mathrm{o} \\
\text { Nov } 1978, \mathrm{t}=3 \mathrm{~m}\end{array}$ & $\begin{array}{ll}2 & (\mathrm{CR}, \mathrm{LW}) \\
3 & \left(\mathrm{VO}_{2}, \mathrm{NH}_{4}, \mathrm{LW}\right)\end{array}$ & Population differences \\
\hline $\begin{array}{l}\mathrm{G} \rightarrow \mathrm{L} \text { vs. GN } \\
\mathrm{G} \rightarrow \mathrm{L} \text { vs. LN } \\
\mathrm{L} \rightarrow \mathrm{G} \text { vs. } \mathrm{LN} \\
\mathrm{L} \rightarrow \mathrm{G} \text { vs. GN }\end{array}$ & $\begin{array}{l}\text { Nov } 1978, t=3 \mathrm{~m} \\
\text { Nov } 1978, t=3 \mathrm{~m} \\
\text { Nov } 1978, t=3 \mathrm{~m} \\
\text { Nov } 1978, t=3 \mathrm{~m}\end{array}$ & $\begin{array}{ll}4 & \left(\mathrm{VO}_{2}, \mathrm{CR}, \mathrm{NH}_{4}, \mathrm{LW}\right) \\
4 & \left(\mathrm{VO}_{2}, \mathrm{CR}, \mathrm{NH}_{4}, \mathrm{LW}\right) \\
2 & \left(\mathrm{NH}_{4}, \mathrm{LW}\right) \\
3 & \left(\mathrm{VO}_{2}, \mathrm{NH}_{4}, \mathrm{LW}\right)\end{array}$ & $\begin{array}{l}\text { Adaptation to Lynher but adaptation not } \\
\text { complete } \\
\text { Adaptation to new environment not complete }\end{array}$ \\
\hline
\end{tabular}

Table 5. Second transplant series. Comparison of native and transplanted Scrobicularia plana at Lynher, E. Looe, and Gannel sites. See legend to Table 4 for details

\begin{tabular}{|c|c|c|c|}
\hline Comparison & Date and time & $\begin{array}{l}\text { List of statistically } \\
\text { significant population } \\
\text { differences in } \\
\text { physiological responses }\end{array}$ & General assessment \\
\hline $\begin{array}{l}\text { LN vs. ELN } \\
\text { LN vs. ELN }\end{array}$ & $\begin{array}{l}\text { Dec 1978, } t=0 \\
\text { Feb 1979, t }=3 \mathrm{~m}\end{array}$ & $\begin{array}{l}4\left(\mathrm{VO}_{2}, \mathrm{CR}, \mathrm{NH}_{4}, \mathrm{LW}\right) \\
1 \text { (CR) }\end{array}$ & Population differences \\
\hline $\begin{array}{l}\mathrm{L} \rightarrow \text { EL vs. } \mathrm{LN} \\
\mathrm{L} \rightarrow \mathrm{EL} \text { vs. ELN } \\
\mathrm{EL} \rightarrow \mathrm{L} \text { vs. ELN } \\
\mathrm{EL} \rightarrow \mathrm{L} \text { vs. LN }\end{array}$ & $\begin{array}{l}\text { Mar 1979, } \mathrm{t}=3 \mathrm{~m} \\
\operatorname{Mar} 1979, \mathrm{t}=3 \mathrm{~m} \\
\operatorname{Mar} 1979, \mathrm{t}=3 \mathrm{~m} \\
\operatorname{Mar} 1979, \mathrm{t}=3 \mathrm{~m}\end{array}$ & $\begin{array}{ll}1 & \left(\mathrm{NH}_{4}\right) \\
2 & \left(\mathrm{VO}_{2}, \mathrm{NH}_{4}\right) \\
2 & \left(\mathrm{CR}, \mathrm{NH}_{4}\right) \\
2 & \left(\mathrm{CR}, \mathrm{NH}_{4}\right)\end{array}$ & $\begin{array}{l}\text { Population differences partly maintained } \\
\text { Partial adaptation to Lymher }\end{array}$ \\
\hline $\begin{array}{l}\text { ELN vs. GN } \\
\text { ELN vs. GN }\end{array}$ & $\begin{array}{l}\text { Dec } 1978, t=o \\
\text { Apr } 1979, t=3 m\end{array}$ & $\begin{array}{l}4\left(\mathrm{VO}_{2}, \mathrm{CR}, \mathrm{NH}_{4}, \mathrm{LW}\right) \\
2\left(\mathrm{VO}_{2}, \mathrm{NH}_{4}\right)\end{array}$ & Population differences \\
\hline $\begin{array}{l}\mathrm{EL} \rightarrow \mathrm{G} \text { vs. ELN } \\
\mathrm{EL} \rightarrow \mathrm{G} \text { vs. GN } \\
\mathrm{G} \rightarrow \mathrm{EL} \text { vs. GN } \\
\mathrm{G} \rightarrow \mathrm{EL} \text { vs. ELN }\end{array}$ & $\begin{array}{l}\text { May } 1979, t=3 \mathrm{~m} \\
\text { May } 1979, \mathrm{t}=3 \mathrm{~m} \\
\text { May 1979,t }=3 \mathrm{~m} \\
\text { May 1979, } \mathrm{t}=3 \mathrm{~m}\end{array}$ & $\begin{array}{ll}2 & (\mathrm{CR}, \mathrm{LW}) \\
1 & (\mathrm{CR}) \\
1 & (\mathrm{CR}) \\
3 & \left(\mathrm{CR}, \mathrm{NH}_{4}, \mathrm{LW}\right)\end{array}$ & $\begin{array}{l}\text { Adaptation to Gannel almost complete } \\
\text { Population differences maintained, little } \\
\text { adaption to E. Looe }\end{array}$ \\
\hline $\begin{array}{l}\text { GN vs. LN } \\
\text { GN vs. LN }\end{array}$ & $\begin{array}{l}\text { Apr 1979, } t=o \\
\text { Jun 1979, } t=3 \mathrm{~m}\end{array}$ & $\begin{array}{l}4\left(\mathrm{VO}_{2}, \mathrm{CR}_{1} \mathrm{NH}_{4}, \mathrm{LW}\right) \\
3\left(\mathrm{CR}, \mathrm{NH}_{4}, \mathrm{LW}\right)\end{array}$ & Population differences \\
\hline $\begin{array}{l}G \rightarrow L \text { vs. GN } \\
G \rightarrow L \text { vs. LN } \\
L \rightarrow G \text { vs. LN } \\
L \rightarrow G \text { vs. GN }\end{array}$ & $\begin{array}{l}\text { Jul } 1979, \mathrm{t}=3 \mathrm{~m} \\
\text { Jul } 1979, \mathrm{t}=3 \mathrm{~m} \\
\text { Jul } 1979, \mathrm{t}=3 \mathrm{~m} \\
\text { Jul } 1979, \mathrm{t}=3 \mathrm{~m}\end{array}$ & $\begin{array}{ll}3 & \left(\mathrm{VO}_{2}, \mathrm{NH}_{4}, \mathrm{LW}\right) \\
1 & \left(\mathrm{NH}_{4}\right) \\
2 & \left(\mathrm{NH}_{4}, \mathrm{LW}\right) \\
1 & (\mathrm{LW})\end{array}$ & $\begin{array}{l}\text { Partial adaptation to Lynher } \\
\text { Adaptation to Gannel almost complete }\end{array}$ \\
\hline
\end{tabular}


presented in Table 6. When Gannel individuals were transplanted to Lynher and E. Looe there was an increase in scope for growth, whereas Lynher individuals transplanted to Gannel showed a decrease in scope for growth. These differences can be explained in terms of site differences in food concentration, particularly chlorophyll $a$, and also differences in clearance rates associated with the different gill areas of the 3 populations. When $S$. plana were transplanted from Lynher to Gannel (August-November 1978) and E. Looe to Gannel (June-September 1978) at a time of spawning and declining food availability, individuals were unable to raise their clearance rates sufficiently to compensate for the lower food levels at the Gannel; consequently, scope for growth declined significantly (Table 6).

Table 6. Scrobicularia plana. Comparison of the scope for growth of native and transplanted individuals from 3 sites. Lynher individuals $=\mathrm{L} ; \mathrm{E}$. Looe $=\mathrm{EL}$; Gannel $=\mathrm{G}$. Transplant symbols, e.g. $\mathrm{G} \rightarrow \mathrm{L}=$ Gannel individuals transplanted to Lynher population. 'Transplant individuals measured in May 1979 were compared with natives measured in April 1979

\begin{tabular}{|c|c|c|}
\hline Population & Date & $\begin{array}{l}\text { Scope for growth } \\
\qquad\left(J d^{-1}\right)\end{array}$ \\
\hline Gannel natives & Nov 1978 & -1.5 \\
\hline Lynher natives & Nov 1978 & +1.9 \\
\hline $\mathrm{G} \rightarrow \mathrm{L}$ & Nov 1978 & +0.9 \\
\hline Gannel natives & - & - \\
\hline Lynher natives & Jun 1979 & +13.0 \\
\hline $\mathrm{G} \rightarrow \mathrm{L}$ & Jul 1979 & +14.4 \\
\hline Gannel natives & Sep 1978 & -0.7 \\
\hline E. Looe natives & Sep 1978 & +0.2 \\
\hline $\mathrm{G} \rightarrow \mathrm{EL}$ & Sep 1978 & +1.3 \\
\hline Lynher natives & Mar 1979 & -2.5 \\
\hline E. Looe natives & Mar 1979 & -1.5 \\
\hline $\mathrm{L} \rightarrow \mathrm{EL}$ & Mar 1979 & -0.6 \\
\hline Gannel natives & Арг 1979 & -3.6 \\
\hline E. Looe natives & Apr 1979 & -0.9 \\
\hline $\mathrm{G} \rightarrow \mathrm{EL}$ & May 1979 & $(+7.2)^{\bullet}$ \\
\hline Lynher natives & Nov 1978 & +3.5 \\
\hline Gannel natives & Nov 1978 & +1.0 \\
\hline $\mathrm{L} \rightarrow \mathrm{G}$ & Nov 1978 & -2.1 \\
\hline E. Looe natives & Apr 1979 & -0.9 \\
\hline Gannel natives & Apr 1979 & -3.6 \\
\hline $\mathrm{EL} \rightarrow \mathrm{G}$ & May 1979 & $(+6.2)^{*}$ \\
\hline
\end{tabular}

The relationship between gill area and tissue weight for the 3 populations of Scrobicularia plana were significantly different (Fig. 1A; Worrall et al., 1983) and when bivalves were transplanted between the 3 sites this relationship showed no reversal or significant alteration towards the native population (Fig. $1 \mathrm{~B}$ and $\mathrm{C}$ ).

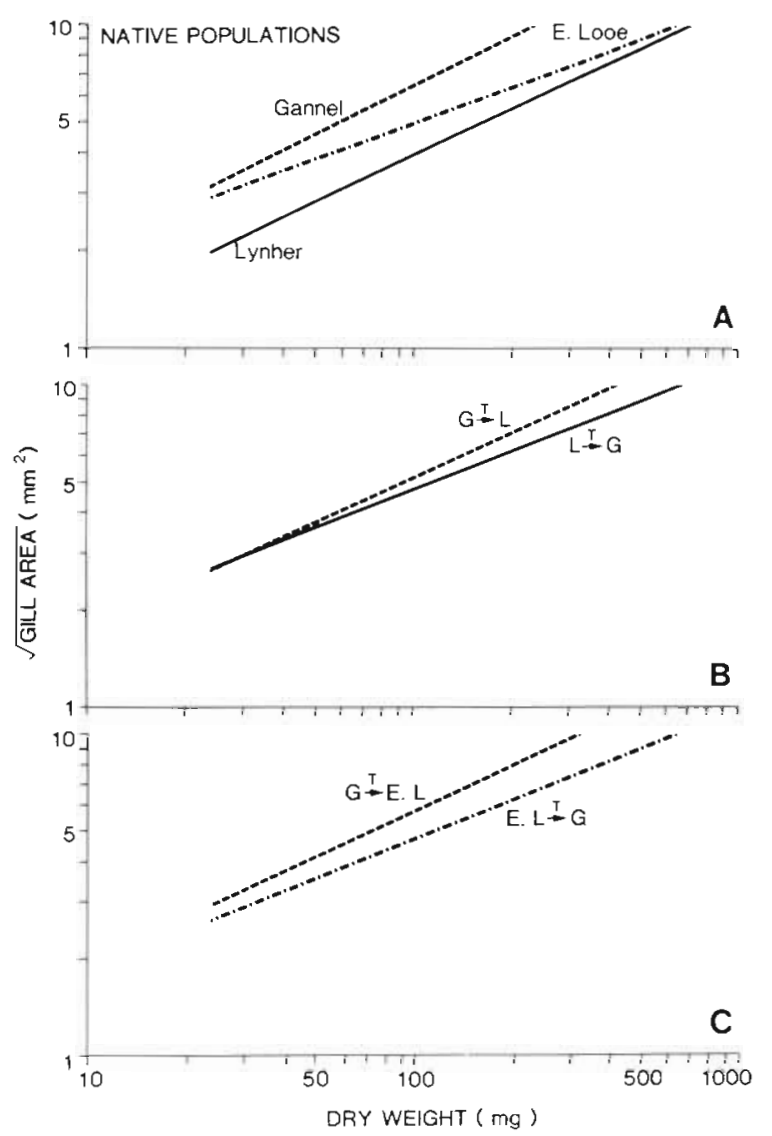

Fig. 1. Scrobicularia plana. Relationship between square root of gill area and dry flesh weight plotted on a logarithmic (base 10) scale. (A) Native populations. Regression equations: Lynher, $\mathrm{Y}=-0.357+0.456(\mathrm{X}),(\mathrm{n}=26, \mathrm{r}=0.98) ; \mathrm{E}$. Looe, $\mathrm{Y}=-0.509+0.371 \quad(\mathrm{X}), \quad(\mathrm{n}=25, \quad \mathrm{r}=0.96) ; \quad$ Gannel, $Y=-0.201+0.504 \quad(X), \quad(n=30, r=0.98)$. (B) Transplants between Lynher and Gannel sites. Regression equations: $\mathrm{L} \rightarrow \mathrm{G}, \quad \mathrm{Y}=-0.115+0.394 \quad(\mathrm{X}), \quad(\mathrm{n}=35, \quad \mathrm{r}=0.88) ; \quad \mathrm{G} \rightarrow \mathrm{L}$, $\mathrm{Y}=-0.220+0.464(\mathrm{X}), \quad(\mathrm{n}=31, \quad \mathrm{r}=0.97)$. (C) Transplants between $\mathrm{E}$. Looe and Gannel sites. Regression equations: $E L \rightarrow G, Y=-0.149+0.408(X),(n=25, \quad r=0.93) ; G \rightarrow E L$, $\mathrm{Y}=-0.187+0.473(\mathrm{X}),(\mathrm{n}=36, \mathrm{r}=0.96) ;$ where $\mathrm{Y}=\log _{10}$ square root gill area $\left(\mathrm{mm}^{2}\right)$ and $X=\log _{10}$ dry flesh weight (mg). Lynher (-), E. Looe (.-.-.-.), Gannel (-- -). Example of transplants, $\mathrm{L} \rightarrow \mathrm{G}=$ Lynher individuals transplanted to Gannel site

The effect of transplantation on the convection requirement varied with the time of year. Scrobicularia plana transplanted from the Gannel to Lynher and E. Looe in the summer (Table 2) showed a marked decline in the convection requirement towards that of the native population. During the winter-spring period, however, bivalves transplanted from Lynher and E. Looe to the Gannel showed a sharp increase (Table 3). There was a loss of relative body weight (measured in terms of $\mathrm{mg}$ tissue per $3-\mathrm{cm}$ individual) and a concomitant decline in $\mathrm{VO}_{2}$ in individuals transplanted to Gannel. Gill area was presumably maintained, thereby resulting in an increase in clearance 
rate per unit weight, and an increased convection requirement. In the reverse situation, when there was an increase in relative weight and $\mathrm{VO}_{2}$ of Gannel individuals transplanted to Lynher and E. Looe there was a decline in clearance rate per unit weight and a decrease in convection requirement. Lynher individuals transplanted to E. Looe (July 1978) showed a decline in relative body weight compared with the Lynher and $\mathrm{E}$. Looe natives, but there was an increase in $\mathrm{VO}_{2}$ which may have been associated with gamete maturation.

Lynher individuals transplanted to Gannel (AugustNovember 1978) showed a loss in relative body weight at a time of reproductive quiescence when food levels in the sediment were low. There was a slight decline in $\mathrm{VO}_{2}$ relative to natives sampled at the start of the transplant (August 1978) but this decline was not significant. At the end of the transplant period (November 1978), the $\mathrm{VO}_{2}$ of the $\mathrm{L} \rightarrow \mathrm{G}$ was significantly higher than of the Gannel natives, and this was accompanied by an enhanced rate of ammonia excretion resulting in a marked decline in the O:N ratio. This low O:N value suggests that protein reserves were being utilized at a time of declining body weight.

The significant differences between native and transplanted populations are summarized in Tables 4 and 5. There was little or only partial adaptation of Gannel and Lynher bivalves on transplantation to the E. Looe site but there was greater adaptation following transplantation of Lynher and E. Looe individuals to the Gannel. Scrobicularia plana transplanted from E. Looe to the Lynher showed almost complete adaptation whereas individuals from Gannel to Lynher showed only partial adaptation.

Physiological responses of transplanted Scrobicularia plana therefore tended to change in the direction of the native population at a particular site, thus indicating that the recorded physiological differences between native populations were largely environmentally induced. The lack of complete, or the slow rate of, acclimatization to a new environment following transplanting is in contrast to the rapid adaptation ( $<6$ wk) shown by Mytilus edulis following reciprocal transplantation between 2 physiologically different mussel populations (Widdows et al., in prep.). The general conclusion of these 2 studies is that observed physiological differences are largely environmentally induced, but the results of the $S$. plana study suggest that when environmental conditions differ markedly or the reproductive state of populations differ, then the acclimatization period may be prolonged.

The major environmental factor affecting the physiology of Scrobicularia plana at the 3 sites is likely to be the particulate food level. The lowest particulate food levels occurred in the Gannel estuary resulting in native and transplanted $S$. plana at this site having a lower body weight and reduced growth rate compared with Lynher and E. Looe individuals. Reproductive condition, an important endogenous factor influencing the physiological condition of bivalves; is dependent on food and temperature. At the Gannel the developmental period was shorter than at the Lynher or E. Looe sites, probably because an adequate food supply was available for a much shorter period of time resulting in more rapid gamete development and culminating in an earlier spawning. Elevated levels of certain heavy metals in the Gannel estuary ( $\mathrm{Pb}, \mathrm{Cd}$ and $\mathrm{Mn}$; Bryan and Hummerstone, 1978; Bryan et al., 1980; Worrall et al., in prep.) may also have contributed to the reduced growth and body size in the Gannel population. The possible influence of high metal levels, in the sediment and body tissues, on the physiology of $S$. plana will be investigated further in a later paper (Worrall et al., in prep.).

Acknowledgements. We are grateful to Dr. B. L. Bayne for helpful discussions and critically reading the manuscript. We are also indebted to Dr. K. R. Clarke for statistical guidance, Mrs. S. Moore for assistance with transplants and to the late Miss C. F. Hawke for the illustrations. This work forms part of the experimental ecology programme of the Institute for Marine Environmental Research, a component of the Natural Environmental Research Council. It was commissioned in part by the Department of the Environment (Contract No. DGR 480/683).

\section{LITERATURE CITED}

Behrens, W. J., Duedall, I. W. (1981). The behaviour of heavy metals in transplanted hard clams, Mercenaria mercenaria. J. Cons. int. Explor. Mer 39: 223-230

Bryan, G. W., Hummerstone, L. G. (1978). Heavy metals in the burrowing bivalve Scrobicularia plana from contaminated and uncontaminated estuaries. J. mar. biol. Ass. U. K. 57 : $401-419$

Bryan, G. W., Langstone, W. J., Hummerstone, L. G. (1980). The use of biological indicators of heavy metal contamination in estuaries with special reference to an assessment of the biological availability of metals in estuarine sediments from south-west Britain. Marine Biological Association of the United Kingdom, Occ. Publ. No. 1

Phelps, D. K., Galloway, W., Thurberg, F. P., Gould, E., Dawson, M. A. (1981). Comparison of several physiological monitoring techniques as applied to the blue mussel, Mytilus edulis, along a gradient of pollutant stress in Narragansett Bay, Rhode Island. In: Vermberg, J, Calabrese, A., Thurberg, F. P., Vernberg, W. B. (ed.) Biological monitoring of marine pollutants. Academic Press, New York, p. 335-355

Simpson, R. D. (1979). Uptake and loss of zinc and lead by mussels (Mytilus edulis) and the relationship with body weight and reproductive cycle. Mar Pollut. Bull. 10: $74-78$ 
Widdows, J., Bayne, B. L., Donkin, P., Livingstone, D. R., Lowe, D. M., Moore, M. N., Salkeld, P. N. (1981a). Measurement of the responses of mussels to environmental stress and pollution in Sullom Voe: a baseline study. Proc. R. Soc. Edinb. 80B: 323-338

Widdows, J., Phelps, D. K., Galloway, W. (1981b). Measurement of physiological condition of mussels transplanted along a pollution gradient in Narragansett Bay. Mar. enviIon. Res. 4: 181-194

Widdows, J., Salkeld, P. N., Cleary, J. J., Donkin, P., Lowe, D. M., Livingstone, D. R., Evans, S. V. (in prep.). Measurement of biological effects and tissue concentrations of some metals and hydrocarbons following reciprocal transplant of mussels (Mytilus edulis L.) between two populations from different environments

Worrall, C. M., Cleary, J. J., Widdows, J., Thomson, P. E. (in prep.) Changes in the tissue metal levels of three native and transplanted populations of Scrobicularia plana (Da Costa) in relation to physiological condition and sediment metal levels

Worrall, C. M., Widdows, J., Lowe, D. M. (1983). Physiological ecology of three populations of the bivalve Scrobicularia plana. Mar Ecol. Prog. Ser. 12: 267--279

This paper was submitted to the editor; it was accepted for printing on February 28, 1983 